\title{
Quantitative analysis of a closed photoacoustic cell that uses a high compliance piezoelectric transducer
}

Jane Hodgkinson

North West Water Ltd, Dawson House, Warrington, WA3 3LW UK, and the Optoelectronics Research Centre, University of Southampton, Southampton, SO17 1BJ UK. Now at Optical Sensors Group, School of Engineering, Cranfield University, Bedfordshire MK43 0AL UK *.

Mark Johnson

North West Water Ltd, Dawson House, Warrington, WA3 3LW UK. Now at Instrumentation Design Ltd, Altrincham, WA14 4QN UK

John P Dakin

Optoelectronics Research Centre, University of Southampton, Southampton, SO17 1BJ UK

\begin{abstract}
A treatment of the operation of low-frequency closed photoacoustic cells is presented, which considers the compliance of various cell elements. The optimum detector, according to our predictions, has a sensor whose compliance is large compared to that of the fluid enclosed by the cell. A simple photoacoustic cell has been built, incorporating a thin-walled piezoelectric cylinder. It was used to test the theory, and demonstrate photoacoustic detection of an absorber in aqueous solution, excited by a low power $678 \mathrm{~nm}$ laser diode modulated at $110 \mathrm{~Hz}$. We have detected absorbances as low as approximately $3 \times 10^{-3} \mathrm{~cm}^{-1}(\mathrm{SNR}=1)$, corresponding to the absorption of light with a modulated power of $50 \mu \mathrm{W}$ rms.
\end{abstract}

PACS numbers: 07.07.Df, 78.20.Hp, 78.30.Cp

\section{Introduction}

There has been much interest in photoacoustic (PA) detection as a sensitive means of determining optical absorption ${ }^{[1,2]}$. Light energy absorbed by an analyte is converted to heat, which causes the analyte and surrounding matrix (gas, liquid or solid) to expand. This expansion may be detected as a transient pressure wave emanating from the source(s) of the absorption, or as a lower frequency increase in the pressure within an enclosed system. The method is considered to have a number of advantages. PA signals are first order measurements of absorption, in that the detected signal in both cases is directly proportional to the light power absorbed, $I_{0^{-}} I$, rather than to that transmitted, $I$. Compared with measurements of the transmitted light $I$, the effects of source flicker noise and shot noise are therefore reduced and the dynamic range of the technique can be large. Additionally, light scattering in the sample has less effect on photoacoustic signals than on conventional measurements, the latter employing effectively smaller acceptance apertures for the transmitted light. 
Much of the recent work on the photoacoustic detection of compounds in liquid solutions has concentrated on the use of pulsed light sources ${ }^{[3]}$. Use of a pulsed source and high speed detection allows measurement of transient photoacoustic waves in open sample containers. The peak incident light power is increased, but this benefit cannot be fully realised in open cells because only a proportion of the transient acoustic wave can be captured by the detector. The approach rules out many $\mathrm{cw}$ light sources that are rugged, reliable and inexpensive, therefore better suited to process environments.

For the analysis of liquid samples, many photoacoustic systems have been designed that employ piezoelectric transducers made of the ceramic lead zirconate titanate (PZT) as acoustic detectors ${ }^{[1]}$. For pulsed operation, the simplest configuration is often a slab of the material, acoustically coupled to the bottom of a cuvette $e^{[4]}$, or contained next to a polished stainless steel probe (which reflects stray light $)^{[3]}$. For low-frequency modulated $\mathrm{cw}$ operation, a closed cell is required to maximise the detected pressure increase and the PZT may form a substantial part of the cell itself. These closed cells therefore make maximal use of the incident light power, an important consideration for low power light sources. Various schemes have been proposed for resonant and non-resonant operation of closed cells $^{[2]}$. The optimum shape for a closed photoacoustic detector is considered to be a compromise between low volume and large optical path length, and therefore should be matched to the shape of the incident light beam ${ }^{[5]}$; cylindrically shaped transducers typically being used with collimated light sources.

Interest in optimising the design of photoacoustic cells comes from two standpoints. Firstly, the signal is proportional to the incident light power and therefore for low power light sources, it is especially necessary to optimise a system's responsivity in order to detect low levels of light absorption. Secondly, recent work on miniaturising the technology fosters interest in design and scaling rules that help drive the development ${ }^{[6]}$. There is, however, little in the way of quantitative prediction of signal levels in closed systems. A quantitative analysis of system performance has recently been successful in describing the performance of closed low frequency (sub resonant) gas cells ${ }^{[6]}$. In such conditions, the bulk compliance of the gaseous analyte dominates over that of other elements such as the sensor and the cell itself. For closed fluid cells where the analyte compliance is much lower, the mechanical compliance of the acoustic sensor and of the cell itself must also be considered.

We present a quantitative analysis, considering the effect of all the significant dc compliances of the cell, including those of the enclosed fluid and of the sensing element. This is valid provided that the modulation frequencies used are much lower than the frequencies of any mechanical resonances in the system. The optimum design is predicted to use a transducer whose mechanical compliance is large compared to that of the enclosed water. The general approach has previously been validated for an alternative photoacoustic / photothermal sensor, based on the use of a water meniscus as a sensor with a high and predictable compliance ${ }^{[7]}$, but to our knowledge the compliance analysis has not been previously applied to PZT detectors.

This paper addresses the operation of a photoacoustic cell based on a thin-walled cylindrical piezoelectric transducer with a high compliance. The response of the device to pressure increases in the cell has been tested, and the system was used to detect trace concentrations of an absorbing compound (potassium permanganate, $\mathrm{KMnO}_{4}$ ) in aqueous solution.

\section{Theory of operation of a simple photoacoustic cell}

Consider a closed cylindrical cell, inside which we assume that the pressure instantaneously reaches a steady-state value whenever a photon is absorbed. This approach is valid provided that we operate at low (sub-resonant) modulation frequencies, for which acoustic wavelengths are much larger than our cell dimensions, the modulation is on a timescale which is shorter than any thermal relaxation in the cell, and the absorber delivers heat energy to the fluid on a timescale shorter than the modulation period. 
Consider a cylindrical cell, with the coordinate system shown in Figure 1. We know that when the cylindrical region of fluid lying in the optical path absorbs a small amount of light energy $\delta E$, its unconstrained volume increase is;

$$
\delta V=\frac{\beta \delta E}{C_{p} \rho}
$$

where $\beta$ is the volume thermal expansion coefficient of the fluid, $C_{p}$ is its specific heat capacity and $\rho$ is its density. Note that, under these conditions, the expression does not involve the original volume of the absorbing region, and we need not make any assumptions about its size, shape or position. Because the temperature increase of the water and the windows will typically be tiny, we can assume that their bulk properties remain unchanged throughout each experiment.

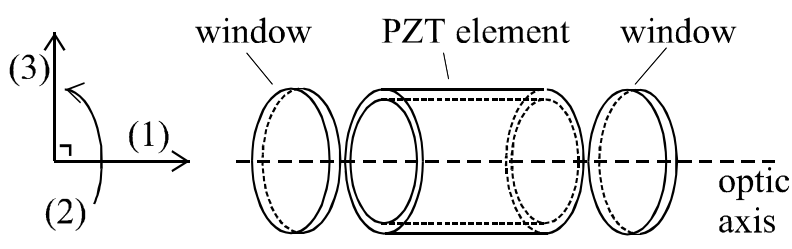

Figure 1. Exploded diagram of a simple photoacoustic cell, showing cylindrical coordinate system.

We wish to calculate the pressure increase in a closed cell due to this energy absorption, by finding the compliance $(\partial V / \partial P)$ of the cell. Firstly, we consider the volume change of different elements in the cell, for a given applied pressure, then find the sum of these quantities. When the pressure inside the cell increases, the water can compress, the piezo can stretch, and the windows can bend. We assume that there are no other significant compliance effects in the cell. Having determined the total compliance, the resulting strain on the piezo can be calculated, and so can the expected magnitude of our signal.

\subsection{Compliance of the PZT}

We can consider the volume expansion of the cylindrical PZT by assuming that it expands by increasing its length and girth rather than by bending. We also assume that the stresses on the piezo in the 1- and 2- directions are separable because there is a net material symmetry between these directions. A net anisotropy exists only in the PZT poling direction, which in this case is the 3direction and is orthogonal to the 1- and 2-directions. Therefore we can consider the stresses in the 1and 2- directions independently, and superimpose their effects.

Firstly consider the effect of an increase in radius. An increase in pressure $\delta \mathrm{P}$ causes a circumferential stress in the walls which must be balanced. The tension in the walls is:

$$
F_{2}=\delta P r_{p} L_{p}=\sigma_{2} t_{p} L_{p}
$$

where $r_{p}$ is the mean (neutral axis) radius of the cylindrical piezo shell, $L_{p}$ is its length, $t_{p}$ is its thickness and $\sigma_{2}$ is the PZT stress in the 2-direction. For the cylinder to increase its enclosed volume, the radius must increase:

$$
\delta V_{2}=2 \pi r_{p} L_{p} \delta r_{p}=2 \pi r_{p}^{2} L_{p} \varepsilon_{2}
$$

where $\varepsilon_{2}$ is the PZT strain in the 2-direction. The Young's modulus for our piezoelectric material, in the circumferential (2) direction, is the same as that in the 1-direction, as explained above, defined as: 


$$
Y_{11}=\frac{\sigma_{1}}{\varepsilon_{1}}=\frac{\sigma_{2}}{\varepsilon_{2}}
$$

Substitution gives the component of the piezo compliance due to expansion in the 2-direction:

$$
\frac{\partial V_{2}}{\partial P}=2 \pi \frac{r_{p}^{3} L_{p}}{t_{p} Y_{11}}
$$

Secondly, we shall consider the effect of an increase in the length of the piezo (expansion in the 1-direction) caused by the pressure increase. The tension force in the piezo walls must balance the pressure over the end of the cylinder:

$$
F_{1}=\pi r_{p}^{2} \delta P=2 \pi r_{p} t_{p} \sigma_{1}
$$

The increase in volume is:

$$
\delta V_{1}=\pi r_{p}^{2} \delta L_{p}=\pi r_{p}^{2} \varepsilon_{1} L_{p}
$$

Substitution gives the compliance due to length increases:

$$
\frac{\partial V_{1}}{\partial P}=\frac{\pi}{2} \frac{r_{p}^{3} L_{p}}{t_{p} Y_{11}}
$$

And the total piezo compliance is the sum of the two compliances in equations (8) and (5).

$$
\frac{\partial V_{p}}{\partial P}=\frac{5 \pi}{2} \frac{r_{p}^{3} L_{p}}{t_{p} \cdot Y_{11}}
$$

\subsection{The compliance of the cell windows and the enclosed water}

The cell windows (see Figure 2) were a potential source of significant unwanted compliance if not designed carefully, because they could bend outwards. They were supported by end couplings with a thin glue line, so we assume that the circular window was effectively constrained in both position and angle around the circumference of its aperture. For these conditions, the deflection $h$ of a thin window under a differential pressure $\delta P$ is given by Timoshenko and Woinowsky-Krieger ${ }^{[8]}$.

$$
h=\frac{12\left(1-v_{\mathrm{w}}^{2}\right)}{64 Y_{\mathrm{w}} t_{\mathrm{w}}^{3}}\left(a^{2}-r^{2}\right)^{2} \delta P
$$

where $v_{\mathrm{w}}$ is the Poisson's ratio for the window, $Y_{\mathrm{w}}$ is its Young's modulus, $t_{\mathrm{w}}$ is its thickness, $a$ is its unsupported radius and $r$ is the radial distance from the 1-axis. Integration gives the total displaced volume $\delta V_{\mathrm{w}}$ for one window:

$$
\delta V_{\mathrm{w}}=\frac{\pi}{16}\left(1-v_{\mathrm{w}}^{2}\right) \frac{a^{6}}{Y_{\mathrm{w}} t_{\mathrm{w}}^{3}} \delta P
$$

So the total compliance for both windows is:

$$
\frac{\partial V_{\mathrm{w}}}{\partial P}=\frac{\pi}{8}\left(1-v_{\mathrm{w}}^{2}\right) \frac{a^{6}}{Y_{\mathrm{w}} t_{\mathrm{w}}^{3}}
$$

The water compliance can be simply determined from the total cell volume, and the definition of the bulk modulus of water, $\kappa$. (equal to $2 \times 10^{9} \mathrm{~Pa}^{[9]}$ ). 


$$
\frac{\partial V_{f}}{\partial P}=\frac{V_{c}}{\kappa}
$$

$\partial V_{f}$ is the volume change due to the fluid expanding, $\partial P$ is the pressure change inside the cell, and $V_{c}$ is the total volume of water inside the cell.

\subsection{The total cell compliance}

If the windows are sufficiently thick, and the cell contains no air bubbles, we may assume that the total compliance of the cell is determined only by the enclosed water and the PZT parameters. Combining the total compliance with equation (1) gives;

$$
\frac{\partial P}{\partial E}=\frac{\beta}{C_{p} \rho_{0}\left(\frac{5 \pi r_{p}^{3} L_{p}}{2 Y_{11} t_{p}}+\frac{V_{c}}{\kappa}\right)}
$$

This enables the response of the piezo to be calculated.

\subsection{PZT response}

The PZT electrodes were attached to the inner and outer surfaces of the cylinder. The stress across the piezo produces a net polarisation, and a current flows from one electrode via a pre-amplifier, putting the PZT into a short-circuit condition. We used a transimpedance amplifier, with feedback resistor $R$, which converted the current output to a voltage signal. The charge on the piezo electrodes, $q$, acts to balance the induced electric field. Our PZT was poled in the 3-direction, so we need the ratio of applied stress in the 1- and 2-directions, to displacement in the 3-direction, which is given by the material constant $d_{31}{ }^{[11]}$ (equal to $d_{32}$ ) and defined as follows:

$$
d_{31}=\frac{q_{1}}{2 \pi r_{p} L_{p} \sigma_{1}}=\frac{q_{2}}{2 \pi r_{p} L_{p} \sigma_{2}}
$$

The total charge $q$ across the piezo is then given by the sum of $q_{1}$ and $q_{2}$, and the voltage output $\Phi$ from the preamplifier is the product of the current and the transimpedance:

$$
\Phi_{\text {out }}=R \frac{\partial q}{\partial t}=\frac{3 \pi R d_{31} r_{p}^{2} L_{p}}{t_{p}} \cdot \frac{\partial P}{\partial t}
$$

Substitution from equation (14) gives the following expression for $\Phi_{\text {out }}$ :

$$
\Phi_{\text {out }}=\frac{6 \pi \beta \kappa R d_{31} Y_{11} L_{p} r_{p}^{2}}{C_{p} \rho\left(2 V_{c} t_{p} Y_{11}+5 \pi \kappa r_{p}^{3} L_{p}\right)} \cdot \frac{\partial E}{\partial t}
$$

Beer's Law states that at any given wavelength $\lambda$, the energy absorbed by the fluid in the cell is given by:

$$
\frac{\partial E}{\partial t}=I_{0}\left(1-10^{-\alpha(\lambda) \ell}\right)
$$

where $I$ is the incident light power, $\ell$ is the optical pathlength in $\mathrm{cm}$ and $\alpha(\lambda)$ is the absorption coefficient of the fluid in $\mathrm{cm}^{-1}$. In fact, $\alpha$ should be considered as the sum of the absorption coefficient of the analyte alone, plus the background absorption coefficient of the water itself. The magnitude of the photoacoustic signal is then given, for small absorption coefficients $(\alpha \ell<<1)$, by: 


$$
\Phi_{\text {out }} \approx \frac{14 \pi \beta \kappa R d_{31} Y_{11} L_{p} r_{p}^{2} \ell}{C_{p} \rho\left(2 V_{c} t_{p} Y_{11}+5 \pi \kappa r_{p}^{3} L_{p}\right)} \alpha I_{0}(t)
$$

This equation may be simplified by introducing a geometrical factor, $\Gamma$, given by the ratio of (i) the water enclosed by the cylindrical PZT $\left(\pi r_{p}{ }^{2} L_{p}\right)$ to (ii) the total volume of water enclosed by the whole cell, including the end couplings $\left(V_{c}\right)$. Its value lies between 0 and 1 , a high value indicating that the cell's end couplings are relatively small.

$$
\Phi_{\text {out }} \approx \frac{14 \beta \kappa R d_{31} Y_{11} \ell \Gamma}{C_{p} \rho\left(2 t_{p} Y_{11}+5 \kappa r_{p} \Gamma\right)} \alpha I_{0}(t)
$$

It should be noted that several of the variables above have a strong dependence on temperature. In particular, the value of $\beta$, and therefore the magnitude of the PA signal, pass through zero at $4{ }^{\circ} \mathrm{C}^{[10]}$.

\section{Optimisation of the photoacoustic cell design}

Equation (19) shows how the photoacoustic signal might be maximised by careful choice of the cell design. Firstly, the optimum piezoelectric element has a wall thickness $t_{p}$ as thin as possible. This is likely to be a practical constraint, because many piezoelectric materials are brittle ceramics and unavailable as very thin elements. Secondly, it is desirable that the PZT should enclose as much of the optical path length as possible, to maximise the detected signal. Dead volume (giving a small value of $\Gamma$ ) decreases the level of the signal because of its bulk compliance, and the analysis here quantifies Rosengren's conclusion that dead volume should be minimised ${ }^{[5]}$. We would also therefore like to reduce the radius of the piezo to a minimum, to reduce the value of $\mathrm{V}_{\mathrm{c}}$. In practice this may be limited by the radius of the incident optical beam, because otherwise stray light might strike the walls of the cell and produce a spurious signal. Finally, a high sensitivity of the piezo to stress, expressed as $d_{3 l}$, is desirable.

For trace analysis, we consider a long optical path length to be desirable, however equation (20) holds different recommendations for different regimes, which we can consider as follows.

(a) Very thin-walled piezoelectric cylinder $\left(2 t_{p} Y_{11}\left\langle\left\langle 5 \kappa r_{p} \Gamma\right)\right.\right.$

In this regime, equation (20) reduces to the following for small $\alpha \ell$.

$$
\Phi_{\text {out }} \approx \frac{14 \beta R d_{31} Y_{11} \ell}{5 C_{p} \rho r_{p}} \alpha I_{0}(t)
$$

Here we can see that a long cell is desirable, regardless of the length of the PZT element, so long as the technical challenge of a low $t_{p}$ can be met. In our case (see section 4 ), this would be satisfied by having a thickness $t_{p}<<0.3 \mathrm{~mm}$, which was difficult to achieve with a free-standing piezo device. 
(b) Thick-walled piezoelectric cylinder $\left.\left.\left(2 t_{p} Y_{11}\right\rangle\right\rangle 5 \kappa r_{p} \Gamma\right)$

For a cylindrical cell, $\Gamma=L_{p} / \ell$ and, again for small $\alpha \ell$,

$$
\Phi_{\text {out }} \approx \frac{14 \beta \kappa R d_{31} L_{p}}{C_{p} \rho 2 t_{p}} \alpha I_{0}(t)
$$

So in this regime, the analysis predicts no benefit from an increase in the full cell length per se, except where that may also lead to an increase in the PZT length $L_{p}$.

(c) Intermediate case.

Our experimental design would actually fall into an intermediate case for which the PZT wall thickness is low, but not as low as we would ideally like. In this case the full analysis using equation (20) is needed to predict the system response.

\section{$4 \quad$ Experimental}

A cylindrical photoacoustic cell was built and evaluated, as shown in Figure 2. The piezoelectric transducer consisted of a cylinder of length $25 \mathrm{~mm}$, radius $6 \mathrm{~mm}$ and thickness $0.79 \mathrm{~mm}$, made of PZT$5 \mathrm{~A}$ which has a high value of the piezoelectric constant $d_{31}=(171 \pm 34) \times 10^{-12} \mathrm{mV}^{-1[11]}$, and was available as a long, thin, thin-walled cylinder. The Young's modulus was $Y_{11}=(6.1 \pm 1.2) \times 10^{10} \mathrm{~Pa}$. The optical path length was $47 \mathrm{~mm}$, giving a cell volume of $5.3 \times 10^{-6} \mathrm{~m}^{3}$. The windows were made from $3 \mathrm{~mm}$ thick fused silica (Suprasil) with $v=0.17^{[12]}$ and $Y=7.31 \times 10^{10} \mathrm{~Pa}^{[9]}$, and had an unsupported radius $a$ of $6 \mathrm{~mm}$. Their compliance was estimated using equation (12) to be negligible in comparison with that of the enclosed water and the PZT. Two end couplings, to permit entry of the sample fluid, were made of $7 \mathrm{~mm}$ thick stainless steel; their compliance was calculated using equation (9), and also found to be negligible. A screw and gasket in one end coupling allowed the cell to be sealed.

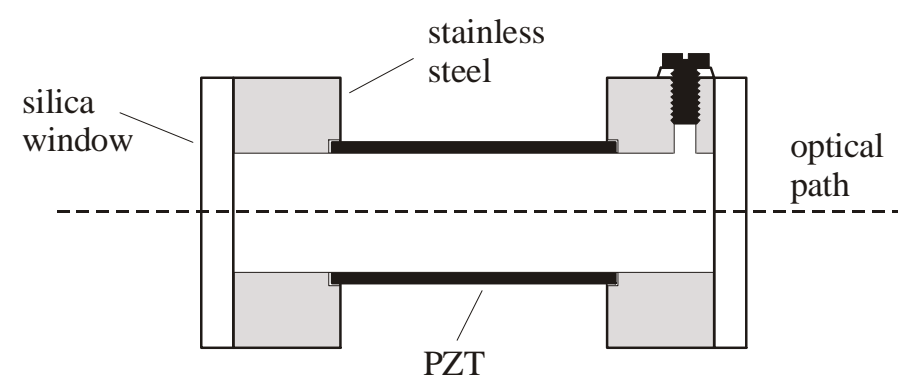

Figure 2. Design of a closed, cylindrical, photoacoustic cell

With this design, we expect the output from the piezo / amplifier to take the following form;

$$
\begin{aligned}
& \Phi_{\text {out }}=(18 \pm 4) \times 10^{-6} \frac{\partial P}{\partial t} \quad \mathrm{volt} \cdot \mathrm{Pa}^{-1} \cdot s \\
& \Phi_{\text {out }}=260 \times 10^{-3} \frac{\partial E}{\partial t} \quad \mathrm{volt} \cdot \mathrm{watt}^{-1}
\end{aligned}
$$

The error in the lower figure is not known. Contributions are likely to arise from $Y_{11}$ and $d_{31}$, each with a quoted $20 \%$ uncertainty ${ }^{[11]}$. However, these two errors are not independent, since each depends on the microstructure of the same PZT, so their combined effect is unknown. 
Figure 3 shows the experimental arrangement of the detection system. The photoacoustic detector was enclosed within an isolation chamber to minimise the effects of environmental acoustic noise. Excitation of the PA signal was achieved using a 678nm laser diode module (RS 194-004), which was square wave modulated using a signal generator (Thurlby Thandar TG220) at a frequency of $110 \mathrm{~Hz}$, corresponding to a region of low noise for photoacoustic detection. The rms modulated power was estimated to be $1.4 \pm 0.2 \mathrm{~mW}$, measured using a large area silicon photodiode (Centronic OST100/7CQ) and pre-amplifier whose responsivity at $678 \mathrm{~nm}$ was determined in the following way. First, we used a fibre pigtailed source at $780 \mathrm{~nm}$ in combination with a commercial, calibrated optical fibre optic power meter (Megger OTP 510), to calibrate the large area photodiode at 780nm. Secondly, we calculated the responsivity of the large area photodiode by using its quoted responsivity curve, relying on repeatability of relative changes along the curve to give the responsivity at $678 \mathrm{~nm}$. Finally we estimated the rms power from the modulated $678 \mathrm{~nm}$ laser diode using the large area photodiode and pre-amplifier in combination with a lock-in amplifier (EG+G 5210) of the type used in subsequent experiments. The measured signal was also recorded on a digital storage oscilloscope (DSO, Hitachi VC-6175) for subsequent analysis.

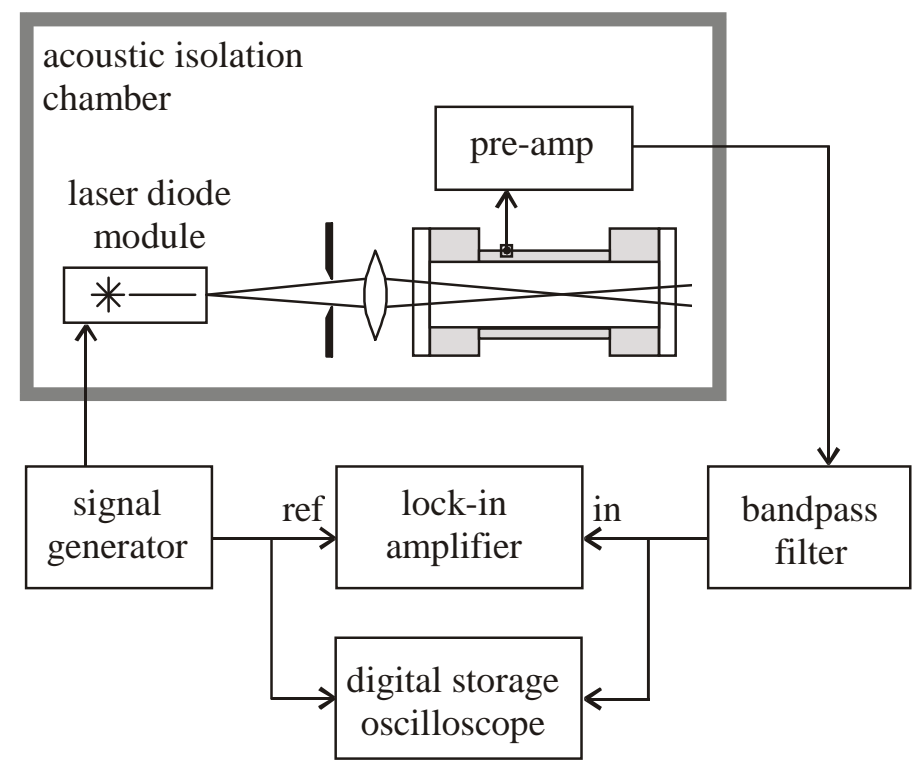

Figure 3. System configuration for photoacoustic detection.

The light was focused into the cell as shown in Figure 3 with a maximum beam diameter at the cell windows of less than $3 \mathrm{~mm}$, and carefully aligned, to minimise the possibility of light striking the walls of the PZT. Stray light was reduced by an aperture, formed by four razor blades, between the laser diode and the lens. The bandpass filter (EG+G 5113) was set to transmit signals between $30 \mathrm{~Hz}$ and $3 \mathrm{kHz}$, with a roll-off of $6 \mathrm{~dB} /$ decade either side of this passband. It also amplified the signal by a factor of 10. Results were recorded by a lock-in amplifier (EG+G 5210) with an integration time constant of 100s, and a digital storage oscilloscope (DSO, Hitachi VC-6175).

The pre-amplifier took the form of a simple transimpedance amplifier, shown in Figure 4. At low frequencies, the PZT was mainly capacitive, with a capacitance of approximately $18 \mathrm{nF}$, and was effectively short-circuited by the pre-amplifier, so that it discharged itself through the feedback resistor, $R$. With the PZT connected to the pre-amplifier, the cell full of water, and electrical and acoustic screening in place, the noise spectrum was determined (using an HP3589A Spectrum analyser) to be approximately $3 \mu \mathrm{V} \mathrm{Hz}^{-1 / 2} \mathrm{rms}$ at $110 \mathrm{~Hz}$; electrical pick-up at this frequency was minimal. 


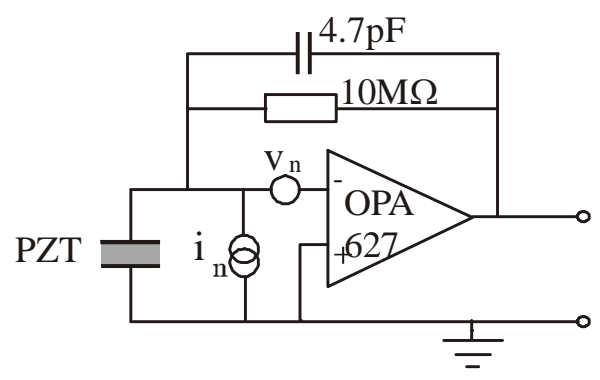

Figure 4. Circuit diagram of the pre-amplifier used for photoacoustic detection.

\subsection{Pressure calibration}

The response of the cell / amplifier to pressure changes was tested experimentally with the cell full of air rather than water. An applied periodic pressure pulse was monitored using a silicon pressure transducer (RS 395-257) and compared to the voltage output from the PZT / pre-amplifier. Figure 5 shows the results as a function of $\partial P / \partial t$, as shown in equation (23), but does not say anything about the cell compliance, $\partial V / \partial P$.

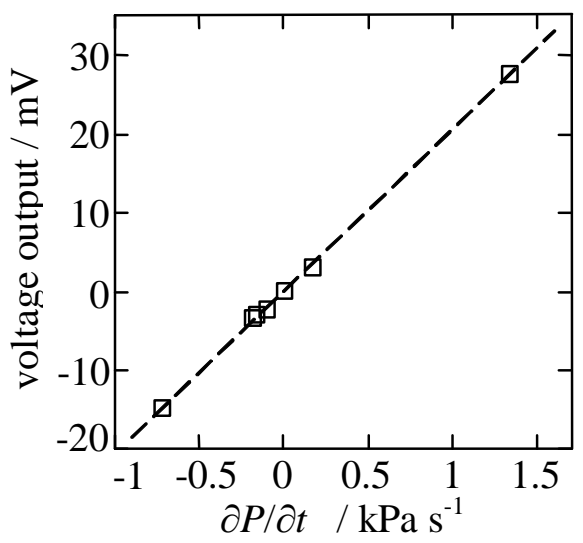

experimental ----- best fit straight line

Figure 5. Calibration plot, showing measured output from the PZT / pre-amplifier versus $\partial P / \partial t$.

\subsection{Photoacoustic detection of an aqueous absorber}

The apparatus described in the preceding sections was used to demonstrate the detection of a photoacoustic signal from an aqueous absorber. The cell was filled with a solution of various concentrations of potassium permanganate $\left(\mathrm{KMnO}_{4}\right)$ in de-ionised water. $\mathrm{KMnO}_{4}$ is known to be a good standard for use as a photoacoustic absorber ${ }^{[13]}$, because it doesn't fluoresce when excited at $678 \mathrm{~nm}$ and delivers heat quickly to the solvent. Its absorption spectrum, measured using a diode array spectrophotometer (HP $8452 \mathrm{~A}$ ) with a $4 \mathrm{~cm}$ pathlength cell, is shown in Figure 6 . The relative emission of the laser diode, measured using the same instrument, has been superimposed. 


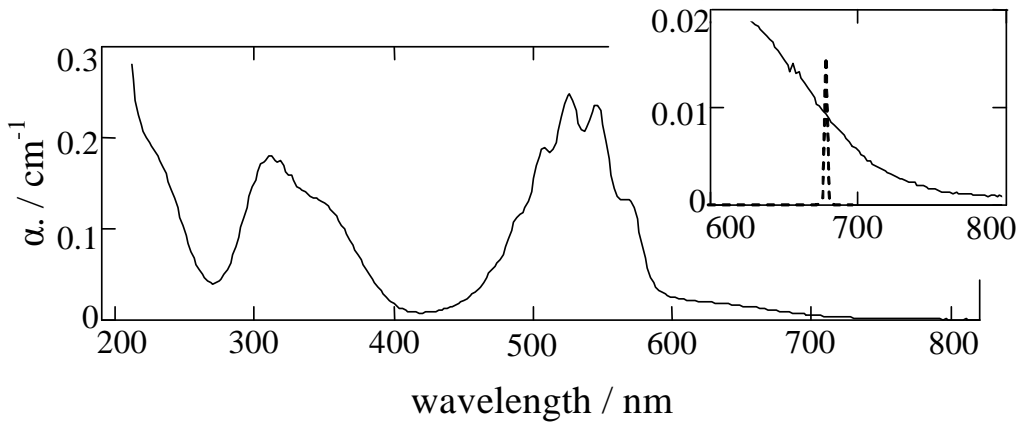

Figure 6. Absorption spectrum of $10^{-4} \mathrm{M} \mathrm{KMnO}_{4}$ in aqueous solution, taken with a $4 \mathrm{~cm}$ pathlength against a deionised water blank. Inset: Emission of red laser diode (arbitrary units, dashed line)

superimposed on the absorption spectrum.

Potassium permanganate solutions were made up by dilution of a standard that consisted of a known dilution of solid $\mathrm{KMnO}_{4}$. The relative concentrations of these solutions were checked by measuring their absorption at $678 \mathrm{~nm}$ using the spectrophotometer. However, for the weakest solutions it was not possible to do this with accuracy and so the absorption at $678 \mathrm{~nm}$ was inferred by measurement at $530 \mathrm{~nm}$, where the absorption was higher. This method was also used to check the stability of the test solutions over the period of experimentation. $\mathrm{KMnO}_{4}$ is known to be unstable in the presence of reducing agents but no evidence was seen for any significant deterioration of our test solutions, to within the limits of the spectrometer, or to within $\pm 3 \%$, whichever was the higher.

The experimental procedure was as follows. The cell was rinsed with the solution to be tested, carefully filled so as not to incorporate any air bubbles, and sealed using the screw and gasket. The acoustic chamber lid was secured, and the measurement could begin. Readings were taken from the lock-in amplifier, once it had stabilised.

\section{Results}

\subsection{Form of the photoacoustic signal}

Figure 7 shows the appearance of one signal recorded by the oscilloscope, averaged over 256 sweeps and further smoothed by averaging over each set of 16 points (the total number of points for each trace was 4000). Also shown is the measured laser diode output, which gives the expected photoacoustic signal, calculated using equations (23) and (18). The shape of the signal is in good agreement with our prediction, but its magnitude is smaller than predicted by a factor of approximately 4 . (The laser diode output was not a perfect square wave, because we were modulating it at a frequency close to its lower limit.) 

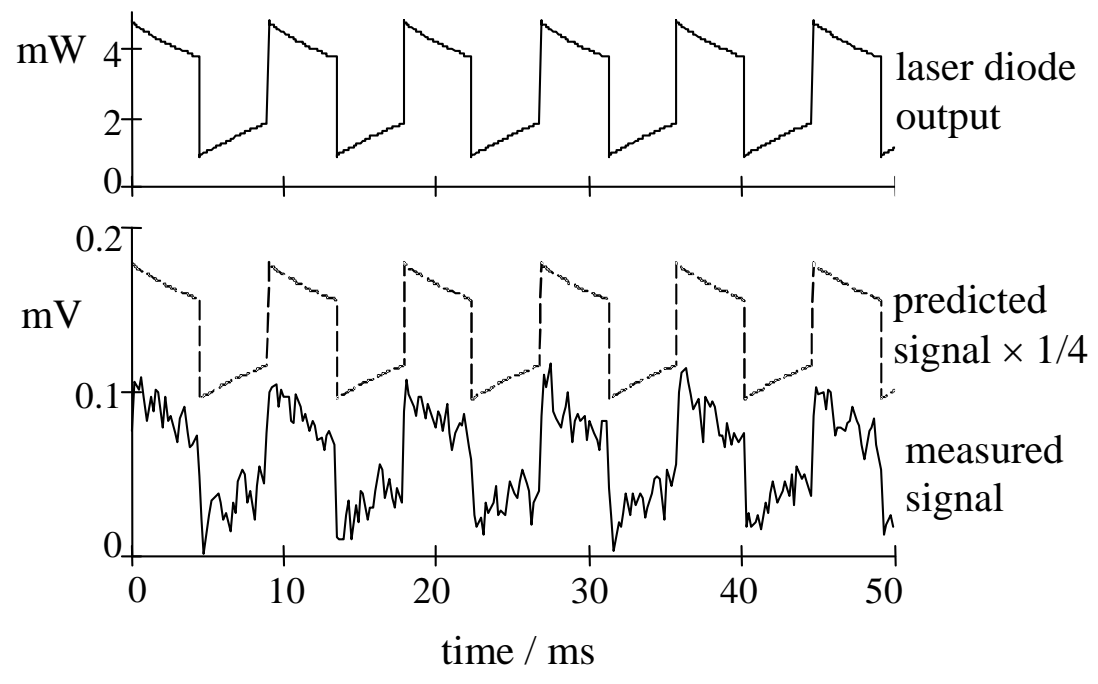

Figure 7. Photoacoustic signals formed by absorption of modulated light in a $3 \times 10^{-4} \mathrm{M}$ solution of $\mathrm{KMnO}_{4}$. The trace shows that the measured signal was approximately four times smaller than predicted

\subsection{Photoacoustic detection of potassium permanganate}

The results of a series of experiments with different concentrations of $\mathrm{KMnO}$, in aqueous solution, are shown in Figure 8, plotted as the magnitude of the output from the PZT / pre-amplifier. We estimated the total level of absorption $\alpha$ of each sample by using the measured absorptions of the $\mathrm{KMnO} 4$ relative to the background, and summing an additional expected signal due to absorption by the water itself (taken to be $4 \times 10^{-3} \mathrm{~cm}^{-1}$ at $678 \mathrm{~nm}^{[3,14]}$ ). The rms modulated power from the laser diode, measured as described in section 4 , was then used to calculate the expected signal given by equations (23) and (18). As expected, Figure 8 (a) shows that the signals reached a maximum value as the absorption coefficient increased, at high absorptions (approaching $\alpha=1$ ). The signals also reached a minimum at the level of the water absorption, as expected. For small absorption coefficients we can subtract the signal due to deionised water alone; this is expected to follow the linear prediction of equation (20) and to improve discrimination of the low absorption coefficients of the analyte itself, as illustrated by Figure 8 (b). 


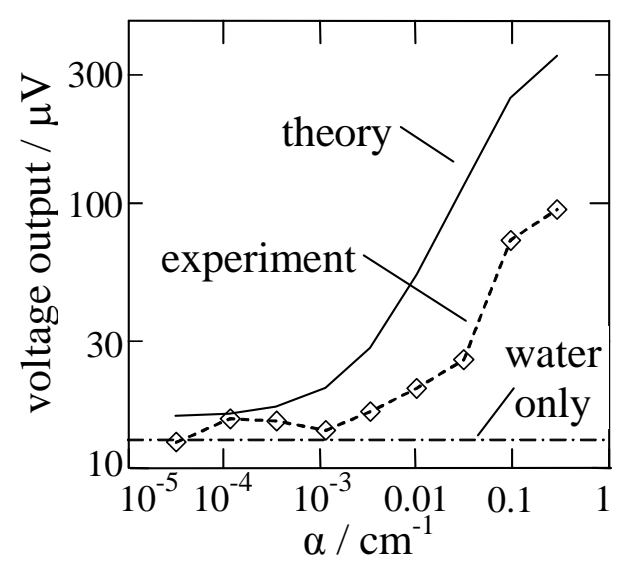

(a)

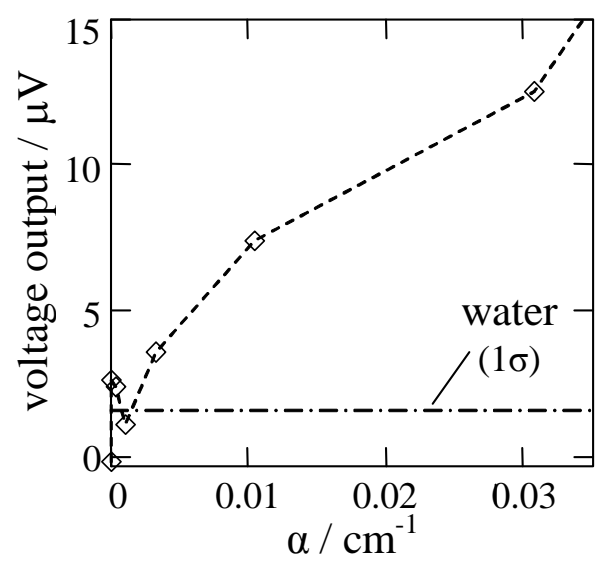

(b)

Figure 8. Output from the PZT / pre-amplifier versus absorption coefficient of $\mathrm{KMnO}_{4}$ alone, (a) raw output, and (b) following subtraction of the signal for deionised water. The trace marked "theory" was calculated using equations (23) and (18).

The results due to $\mathrm{KMnO}_{4}$ absorption were smaller than expected by a factor of approximately 4 . There are a number of possible explanations. Although some of the reduction is attributable to the large error in the predicted responsivity resulting from uncertainty in the material constants in the PZT, this is insufficient to account for much of the error. It is suggested that the entire system might not be as efficient as expected, because of a higher cell compliance than predicted, which could have resulted from two effects that were not modelled. It is unlikely that either of these effects could have been readily avoided in the present design. It is possible (i) that the PZT element were able to bow outwards slightly in the middle, allowing it to increase its compliance without a compensating increase in signal levels, and / or (ii) that the adhesive joints holding it to the stainless steel end couplings were able to flex, contributing a second unquantified compliance that could have reduced signal levels. These latter joints were necessarily made of a strong, waterproof and electrically insulating material (epoxy resin) that happened to be more flexible than the other cell materials. The PZT cylinder had been placed in small recesses in the end coupling, with the adhesive used to fill the gaps such that the resulting bond line was approximately $0.5 \mathrm{~mm}$ wide (radial direction) $\times 1 \mathrm{~mm}$ deep (longitudinal direction).

The asymptotic value of the PA signals at low levels of $\mathrm{KMnO}_{4}$, ie the signal due to water absorption alone, was approximately at the predicted value. However, if the system responsivity is lower than expected, this asymptote would not be the result of water absorption, as expected, but of some other effect such as window noise ${ }^{[2]}$, stray light hitting the cell walls, or environmental acoustic noise. (The expected level of noise from the detector is small in comparison with these results, being approximately $0.3 \mu \mathrm{V}$ rms for an integration period of 100s.) The smallest detectable absorption coefficient was approximately $3 \times 10^{-3} \mathrm{~cm}^{-1}(\mathrm{SNR}=1)$. This would not presently offer improved detection limits over straightforward spectrophotometry ${ }^{[15]}$; further advantages are needed to make PA detection attractive, such as its known tolerance to light scattering in turbid samples. However, for a photoacoustic technique the detection limit represents efficient use of low levels of incident power, corresponding to the absorption of light with an rms modulated power of $50 \mu \mathrm{W}$.

Equations (20) and (21), if valid, indicate the potential for improvement in the signal to noise ratio for an ideally thin transducer whose properties are otherwise unchanged. The limit of improvement for thinner transducers is by a factor of 4.5 on this analysis. 
A simple closed, cylindrical photoacoustic cell has been designed, built, and used to demonstrate photoacoustic detection of an absorber in aqueous solution, excited by continuous-wave sources, modulated at relatively low frequencies. For low power light sources such as laser diodes, it was particularly important that the responsivity of the cell was optimised. To this end, a theory of the operation of the cell has been developed, tested, and used to optimise the cell design, within the limits of practicality.

The ideal detector, according to our predictions, has a sensor whose compliance is large compared to that of the water enclosed by the cell. For our cylinder of PZT-5A, this would have required a wall thickness of $t_{p}<<0.3 \mathrm{~mm}$. Instead, using the thinnest commercially available PZT element with a wall thickness of $0.79 \mathrm{~mm}$, and potassium permanganate in aqueous solution as a standard absorber, we have demonstrated photoacoustic signals that were a factor of four smaller than our predictions, for the most strongly absorbing solutions. It is suggested that the cell compliance may have been greater than we estimated, which would have reduced the signal level. The minimum detectable absorbance of the system was approximately $3 \times 10^{-3} \mathrm{~cm}^{-1}$, corresponding to the absorption of light with a modulated power of only $50 \mu \mathrm{W}$ rms.

\section{Acknowledgements}

The work was supported by North West Water Ltd and by the Department of Trade and Industry, under the Teaching Company Scheme.

\section{References}

[1] A. Rosencwaig. Photoacoustics and Photoacoustic Spectroscopy (Wiley, New York, 1980)

[2] Y.-H. Pao. Optoacoustic Spectroscopy and Detection. (Academic Press, New York, 1977)

[3] C. K. N. Patel and A. C.Tam, Rev. Mod. Phys. 53, 517 (1981).

[4] E. Voigtman, A. Jurgensen and J. Wineforder, Anal. Chem. 53, 1442 (1981).

[5] L.-G. Rosengren, Appl. Opt. 14, 1960 (1975).

[6] S. L. Firebaugh, K. F. Jensen and M. A. Schmidt, J. Appl. Phys. 92 (3) 1555 (2002)

[7] J. Hodgkinson, M. Johnson and J. P. Dakin, Meas. Sci. Tech. 9, 1316 (1998)

[8] S. Timoshenko and S. Woinowsky-Krieger, Theory of plates and shells (McGraw-Hill, New York, 1959)

[9] Kaye G W C and Laby T H. Tables of Physical and Chemical Constants. (Longmans, London, 13th edition 1966)

[10] M. W. Sigrist. J. Appl. Phys., 60, 7, pp R83-R121 (1986)

[11] Technical data sheet for PZT transducers (Morgan Matroc Ltd, Thornhill, Southampton, 1995). 
[12] Lide D R (Editor). Handbook of Chemistry and Physics, CRC Press, 73rd edition (1992)

[13] S. E. Braslavsky and G. E. Heibel, Chem. Rev. 92, 1381 (1992)

[14] G. M. Hale and M. R. Querry. Appl. Opt. 12, 555-563 (1973)

[15] F Manoochehri and E Ikonen. Appl. Opt. 34, 3686-3692 (1995) 
